\title{
ASSOCIAÇÃO ENTRE A ADESÃO À DIETA MEDITERRÂNICA E A QUALIDADE DE VIDA DE PACIENTES DIABÉTICOS
}

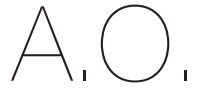
ARTIGO ORIGINAL

${ }^{1}$ Unidade de Nutrição do Hospital Distrital de Santarém,

Avenida Bernardo

Santareno, n. ${ }^{\circ} 3737$ B,

2005-177 Santarém

Portugal

*Endereço para correspondência:

Mário Rui Abade

Rua da Teresa, n. 15

2125-032 Glória do Ribatejo

Portugal

marioruiabade@outlook.pt

Histórico do artigo:

Recebido a 29 de janeiro de 2019 Aceite a 24 de setembro de 2019

\author{
RELATIONSHIP BETWEEN ADHERENCE TO THE \\ MEDITERRANEAN DIET AND QUALITY OF LIFE OF DIABETIC \\ PATIENTS
}

Mário Rui Abade ${ }^{1 *}$; José de Santo Amaro

\section{RESUMO}

INTRODUÇÃO: Na população diabética, a adesão à Dieta Mediterrânica está associada a uma redução das complicações médicas, permitindo um melhor controlo glicémico e do peso corporal. No entanto, são escassos os estudos que avaliem se uma boa adesão à Dieta Mediterrânica se traduz numa melhor Qualidade de Vida Relacionada com a Saúde dos indivíduos diabéticos.

OBJETIVos: Este estudo pretende estudar a associação entre a adesão à Dieta Mediterrânica e Qualidade de Vida Relacionada com a Saúde em pacientes com diabetes tipo 2.

METOdologiA: Avaliou-se a Qualidade de Vida Relacionada com a Saúde de 185 pacientes (103 mulheres e 82 homens) através do preenchimento de um instrumento genérico (SF-36), e um específico para a diabetes, o Perfil de Saúde do Diabético (DHP-18). Os participantes foram entrevistados para preenchimento de uma ferramenta de avaliação de adesão à Dieta Mediterrânica, 0 PREDIMED. Para efetuar a análise estatística utilizou-se o programa SPSS. Todos os testes estatísticos foram efetuados com um intervalo de confiança de 95\%, considerando um valor de $p<0,05$, estatisticamente significativo.

RESULTADOS: A amostra apresenta uma elevada prevalência de obesidade (43,7\%), dislipidemia (77,8\%), hipertensão (87,4\%), e valores de hemoglobina glicada (77,2\%) acima do recomendado, no grupo de indivíduos com fraca adesão à Dieta Mediterrânica, comparativamente ao grupo com boa adesão. O score de adesão à Dieta Mediterrânica está significativamente associado a scores mais elevados ( $p<0,05)$ em algumas dimensões do SF-36: saúde geral, função física, desempenho físico, assim como, na medida sumário física.

CONCLUSõES: As pessoas com diabetes tipo 2 têm uma melhor Qualidade de Vida Relacionada com a Saúde sobretudo nas dimensões da saúde física, se recorrerem a uma dieta baseada nos princípios da Dieta Mediterrânica. Seria importante desenvolver programas de educação para a saúde que promovam a adoção da Dieta Mediterrânica tanto a nível individual como comunitário entre a população diabética, como estratégia de redução de carga de doença, e prevenção ou retardamento das complicações associadas à diabetes, que poderão originar perda de funcionalidade e/ou incapacidade física.

\section{PALAVRAS-CHAVE}

Adesão à Dieta Mediterrânica, Diabetes tipo 2, Qualidade de Vida Relacionada com a Saúde

ABSTRACT

INTRODUCTION: In diabetic population, the adherence to Mediterranean Diet is associated with fewer medical complications, which allows a better glycemic and weight control. However, there is a lack of studies that assesses if good adherence to Mediterranean Diet results in better Health-Related Quality of Life in diabetic individuals.

OBJECTIVES: This study intends to assess the association between adherence to Mediterranean Diet and Health-Related Quality of Life in patients with type 2 diabetes.

METHODOLOGY: We assessed Health-Related Quality of Life in 185 patients (103 women and 82 men) by filling a generic questionnaire (SF-36) and a specific one for diabetes, the Diabetes Health Profile (DHP-18). The participants were individually interviewed to complete an assessment tool of the adherence to Mediterranean Diet, the PREDIMED. Statistical analysis was conducted with SPSS. All statistical tests were performed with a $95 \%$ confidence interval, considering a p-value of $<0.05$ statistically significant. RESULTS: Our sample shows a higher prevalence of obesity (43.7\%), dyslipidemia (77.8\%), hypertension (87.4\%), and glycated hemoglobin (77.2\%) over the recommendation, in the poor adherence to Mediterranean Diet group comparing to good adherence group. The adherence to Mediterranean Diet score is significantly associated with higher scores $(p<0.05)$ in some SF-36 dimensions: general health, physical functioning, role functioning, as well, in the physical summary measure. ConcLusıons: People with type 2 diabetes have better Health-Related Quality of Life especially in physical health dimensions by appealing to a diet based on the principles of Mediterranean Diet. It will be important to develop health education programs that promote the adoption of Mediterranean Diet in diabetic population at either individual and community level, as well a strategy to reduce the disease burden, and prevent or delay diabetes-related complications that may cause loss of functionality and/or physical disability.

KEYWORDS

Adherence to Mediterranean Diet, Type 2 diabetes, Health-Related Quality of Life 


\section{INTRODUÇÃO}

A Dieta Mediterrânica (DMed) traduz o padrão alimentar dos países banhados pelo Mar Mediterrânico ou que por ele são influenciados (1). Esta dieta assenta numa base sociogeográfica, cultural e de sustentabilidade, que privilegia a utilização de alimentos tradicionais e da época, confecionados através de práticas culinárias simples e frugais. Este padrão alimentar caracteriza-se por um consumo elevado de alimentos de origem vegetal (cereais integrais, hortofrutícolas, leguminosas, frutos oleaginosos, sementes e azeitonas) e azeite como gordura de eleição. E, por outro lado, por um consumo moderado de pescado, marisco, ovos, carne de aves, produtos lácteos (queijo e iogurte) e bebidas alcoólicas (sobretudo vinho tinto para acompanhar as refeições principais), assim como, um baixo consumo de carnes vermelhas. Na nova pirâmide da DMed salienta-se ainda a importância da convivialidade à volta da mesa e da prática regular de atividade física (2). Uma boa adesão a este padrão alimentar contribui para o aumento da longevidade, diminuição da morbilidade e mortalidade por doenças cardiovasculares e cancro, diminuição da incidência de obesidade, diabetes mellitus tipo 2 (DM2) e hipertensão, e, por conseguinte, para uma melhor qualidade de vida (3).

A DM2 é uma patologia crónica caracterizada por uma alteração do metabolismo dos hidratos de carbono associada à insulinorresistência que resulta no aumento dos níveis de glicemia. Segundo os dados epidemiológicos mais recentes, em 2015, esta condição afetava cerca de $13,3 \%$ da população portuguesa com idades compreendidas entre os 20 e os 79 anos (4).

A Qualidade de Vida Relacionada com a Saúde (QdVRS) pode ser definida como uma avaliação subjetiva e multidimensional que um indivíduo faz sobre a sua própria saúde, bem-estar, e o impacto da sua doença nas componentes físicas, psicológicas, emocionais e sociais (5, 6). Sendo a DM2 uma doença com atingimento a longo prazo de vários órgãos e sistemas, esta apresenta implicações importantes na qualidade de vida dos doentes, tanto pelas complicações médicas como também pelo estigma social que a doença ainda representa (7).

Em 2016, um estudo publicado por Alcubierre et al., forneceu-nos resultados preliminares sugestivos sobre uma possível associação positiva entre a adesão à DMed e a qualidade de vida de doentes com DM2 (8).

\section{OBJETIVOS}

Com este estudo pretendemos caracterizar o consumo alimentar da amostra, segundo as recomendações da DMed; identificar o nível de adesão à DMed da amostra; e estudar a relação entre a adesão à DMed e a QdVRS de pacientes com DM2.

\section{METODOLOGIA}

Este é um estudo observacional e transversal. A amostra é constituída por 185 pacientes (103 mulheres e 82 homens) com DM2 e idade igual ou superior a 18 anos, recrutados entre os dias 29 de maio e 31 de outubro de 2018. Foram excluídos da amostra os indivíduos: com análises bioquímicas com validade superior a três meses; imobilizados; com histórico de internamento, acidentes, quedas ou fraturas ósseas no último mês; e aqueles dependentes nas suas atividades diárias. Isto serviu para controlar outros fatores que podem influenciar a QdVRS reportada, além da adesão à DMed. Mediu-se o peso corporal dos participantes utilizando uma balança, aplicando o protocolo constante na Orientação da Direção-Geral da Saúde n. ${ }^{\circ}$ 17/2013 - Avaliação Antropométrica no Adulto (9). Para calcular o índice de massa corporal (IMC) utilizou-se o valor da estatura contida no documento de identificação civil de cada indivíduo, por não haver estadiómetro disponível em todos os gabinetes de avaliação. Identificou-se o score de adesão à DMed através da aplicação de protocolo PREDIMED (PREvención com Dieta MEDiterránea) composto por 14 perguntas (10). Considera-se uma boa adesão se o valor do score obtido no inquérito for $\geq 10$ pontos (11). Avaliou-se a QdVRS através da aplicação de um instrumento de medição genérico composto por 36 perguntas (SF-36), que permite ao entrevistado avaliar o seu estado de saúde em oito dimensões: saúde geral (SG), desempenho físico (DF), função física (FF), dor física (DR), desempenho emocional (DE), função social (FS), vitalidade (VT), e saúde mental (SM). Estas dimensões podem ser ainda agregadas de forma a obter-se as medidas sumário física (SG, DF, FF e DR) e mental (DE, FS, VT e SM) (12). Administrou-se também um instrumento específico para avaliar a QdVRS na população diabética, o Perfil de Saúde do Diabético (Diabetes Health Profile - DHP-18). Ao contrário do SF-36, nas dimensões do DHP-18 (sofrimento psicológico, barreiras à atividade e alimentação desinibida) as pontuações são apresentadas numa escala de orientação negativa em que quanto maior for o valor, pior é a perceção da qualidade de vida (13). As respostas dos questionários foram informatizadas e transformadas em escalas de 0 a 100, conforme proposto pelos autores que os validaram para uso na população portuguesa (12-15). Todos os questionários foram aplicados de forma indireta, através de entrevista presencial. A análise estatística foi realizada utilizando o programa SPSS, versão 23. Previamente à realização dos testes estatísticos os "outliers" foram removidos segundo a aplicação do teste de Tukey. Efetuou-se uma análise descritiva das variáveis por categoria de adesão à DMed (boa ou fraca). Analisou-se a relação entre a adesão à DMed e as várias dimensões dos questionários de avaliação da QdVRS, efetuando uma análise de regressão linear simples e múltipla. Nesta última, também se incluíram no modelo os seguintes fatores: sociodemográficos (idade, género, estado civil, nível de escolaridade), sobre o estilo de vida (hábitos tabágicos e de atividade física), estado nutricional (IMC - variável contínua), histórico de saúde (dislipidemia e/ou hipertensão - variáveis dicotómicas), e bioquímicos (hemoglobina glicada - A1c - valor de referência $<7,0 \%$ ). Antes da realização da análise de regressão linear conferiu-se a normalidade da distribuição dos resíduos, e, a sua independência pelo teste de Durbin-Watson. A ausência de multicolinearidade foi verificada pelo fator de inflação da variância (VIF), e a homocedasticidade pela análise do diagrama dos resíduos. O protocolo do estudo foi aprovado pela Comissão de Ética do Hospital Distrital de Santarém.

\section{RESULTADOS}

A idade média da amostra é de $67 \pm 10$ anos, a idade mínima 38 anos, e a máxima 92 anos. Foram avaliadas 103 mulheres, das quais $9(8,7 \%)$ têm boa adesão à DMed e $94(91,3 \%)$ têm fraca adesão; relativamente aos homens, 9 (11,0\%) têm boa adesão e 73 (89,0\%) têm fraca adesão. Há que salientar que 90,3\% ( $n=167)$ dos inquiridos têm uma fraca adesão à DMed, enquanto que, apenas 9,7\% ( $n=18)$ parecem aderir a este padrão alimentar. O IMC médio é de 26,9 \pm 3,3 $\mathrm{kg} / \mathrm{m}^{2}$ e 30,0 $\pm 5,4 \mathrm{~kg} / \mathrm{m}^{2}$, nos grupos de boa e fraca adesão à DMed, respetivamente. Verifica-se uma prevalência elevada de indivíduos classificados com obesidade (43,7\%) no grupo com fraca adesão à DMed comparativamente ao grupo com boa adesão (11,1\%). Já os valores de A1c estão predominantemente acima do valor de referência no grupo de indivíduos com fraca adesão $(77,2 \%)$ quando comparado com o grupo com boa adesão (72,2\%). Para além disso, a prevalência de dislipidemia $(77,8 \%)$ e hipertensão $(87,4 \%)$ é superior no grupo de indivíduos com fraca adesão à DMed, comparativamente ao grupo com boa adesão (Tabela 1). De acordo com os dados obtidos com o PREDIMED, a amostra apresenta uma baixa prevalência de consumo de 
frutas (44,9\%), leguminosas (31,9\%), azeite (13,0\%) e frutos oleaginosos (10,8\%), e, por outro lado, um consumo elevado de manteiga, margarina ou natas (59,5\%) (Tabela 2). Os pacientes com boa adesão à DMed têm significativamente scores mais elevados $(p<0,05)$ em algumas dimensões do SF-36: SG, FF, DF, assim como, na medida sumário física (SG, DF, FF e DR), mesmo depois de ajustados para a idade, género, IMC, A1c, atividade física, hábitos tabágicos, hipertensão, dislipidemia, nível de escolaridade e estado civil. Na dimensão da SM encontrou-se um valor estatisticamente significativo apenas no modelo não ajustado. Pelo contrário, uma boa adesão à DMed está associada a scores mais baixos numa das dimensões do DHP-18 (barreiras à atividade), o que não se verificou no modelo ajustado para outros fatores que influenciam a qualidade de vida além da adesão à DMed (Tabela 3).

Tabela 1

Caracterização da amostra por adesão à Dieta Mediterrânica

\begin{tabular}{|c|c|c|c|c|c|}
\hline \multirow{2}{*}{ CARACTERÍSTICA } & \multirow{2}{*}{ CATEGORIA } & \multicolumn{2}{|c|}{ BOA ADESÃO DMED ( $n=18)$} & \multicolumn{2}{|c|}{ FRACA ADESÃO DMED ( $n=167$} \\
\hline & & $n$ & $\%$ & $\mathrm{n}$ & $\%$ \\
\hline \multirow{2}{*}{ Género } & Homens & 9 & 50,0 & 73 & 43,7 \\
\hline & Mulheres & 9 & 50,0 & 94 & 56,3 \\
\hline \multirow{4}{*}{ IMCa } & Baixo peso & 0 & 0,0 & 1 & 0,6 \\
\hline & Peso adequado & 6 & 33,3 & 25 & 15,0 \\
\hline & Pré-obesidade & 10 & 55,6 & 68 & 40,7 \\
\hline & Obesidade & 2 & 11,1 & 73 & 43,7 \\
\hline \multirow{2}{*}{ Atividade física ${ }^{a}$} & $\begin{array}{l}\text { Sedentário } \\
(<150 \text { minutos por semana) }\end{array}$ & 17 & 94,4 & 137 & 82,0 \\
\hline & $\begin{array}{l}\text { Ativo } \\
(\geq 150 \text { minutos por semana) }\end{array}$ & 1 & 5,6 & 30 & 18,0 \\
\hline \multirow{2}{*}{ Hábitos tabágicos } & Fumadores & 4 & 22,2 & 10 & 6,0 \\
\hline & Não fumadores & 14 & 77,8 & 157 & 94,0 \\
\hline \multirow{2}{*}{$A 1 c^{b}$} & $<7,0 \%$ & 5 & 27,8 & 38 & 22,8 \\
\hline & $\geq 7,0 \%$ & 13 & 72,2 & 129 & 77,2 \\
\hline \multirow{2}{*}{ Dislipidemia } & Sim & 13 & 72,2 & 130 & 77,8 \\
\hline & Não & 5 & 27,8 & 37 & 22,2 \\
\hline \multirow{2}{*}{ Hipertensão } & Sim & 11 & 61,1 & 146 & 87,4 \\
\hline & Não & 7 & 38,9 & 21 & 12,6 \\
\hline \multirow{3}{*}{ Nível de escolaridade } & Ensino básico ou menos & 15 & 83,3 & 140 & 83,8 \\
\hline & Ensino secundário & 3 & 16,7 & 19 & 11,4 \\
\hline & Ensino superior & 0 & 0,0 & 8 & 4,8 \\
\hline \multirow{4}{*}{ Estado civil } & Solteiro & 0 & 0,0 & 10 & 6,0 \\
\hline & Casado & 15 & 83,3 & 114 & 68,2 \\
\hline & Divorciado & 1 & 5,6 & 15 & 9,0 \\
\hline & Viúvo & 2 & 11,1 & 28 & 16,8 \\
\hline $\begin{array}{l}\text { a Organização Mundial da Saúde } \\
\text { ' American Diabetes Association }\end{array}$ & $\begin{array}{l}\text { A1c: Hem } \\
\text { Adesão D }\end{array}$ & Mec & & Massa $C$ & \\
\hline
\end{tabular}

Descrição das questões de adesão à Dieta Mediterrânica

\begin{tabular}{|c|c|c|c|}
\hline QUESTÕES DE ADESÃO À DIETA MEDITERRÂNICA & CRITÉRIO PARA 1 PONTO & O PONTOS (\%) & 1 PONTO (\%) \\
\hline 1. Utiliza azeite como principal gordura culinária? & Sim & 1,6 & 98,4 \\
\hline $\begin{array}{l}\text { 2. Que quantidade de azeite consome num dia (incluindo uso para fritar, temperar saladas, } \\
\text { refeições fora de casa, etc.)? }\end{array}$ & $\geq 4$ colheres de sopa & 87,0 & 13,0 \\
\hline $\begin{array}{l}\text { 3. Quantas porções de produtos hortícolas consome por dia? (1 porção: } 200 \mathrm{~g} \text {; considere } \\
\text { acompanhamentos como metade de uma porção) }\end{array}$ & $\begin{array}{l}\geq 2 \text { ( } \geq 1 \text { se for porção em cru } \\
\text { ou em salada) }\end{array}$ & 35,7 & 64,3 \\
\hline 4. Quantas peças de fruta (incluindo sumos de fruta natural) consome por dia? & $\geq 3$ & 55,1 & 44,9 \\
\hline $\begin{array}{l}\text { 5. Quantas porções de carne vermelha, hambúrguer ou produtos cárneos (presunto, salsicha, } \\
\text { etc.) consome por dia? (1 porção: } 100-150 \mathrm{~g} \text { ) }\end{array}$ & $<1$ & 27,6 & 72,4 \\
\hline 6. Quantas porções de manteiga, margarina, ou natas consome por dia? (1 porção: 12 g) & $<1$ & 59,5 & 40,5 \\
\hline 7. Quantas bebidas açucaradas ou gaseificadas bebe por dia? & $<1$ & 9,7 & 90,3 \\
\hline 8. Quantos copos de vinho bebe por semana? & $\geq 7$ copos & 75,1 & 24,9 \\
\hline 9. Quantas porções de leguminosas consome por semana? (1 porção: $150 \mathrm{~g}$ ) & $\geq 3$ & 68,1 & 31,9 \\
\hline $\begin{array}{l}\text { 10. Quantas porções de peixe ou marisco consome por semana? (1 porção: 100-150 g de peixe } \\
\text { ou } 4-5 \text { unidades ou } 200 \mathrm{~g} \text { de marisco) }\end{array}$ & $\geq 3$ & 20,0 & 80,0 \\
\hline $\begin{array}{l}\text { 11. Quantas vezes por semana consome produtos de pastelaria ou doces comerciais (não } \\
\text { caseiros), como bolos, bolachas, biscoitos? }\end{array}$ & $<3$ & 54,1 & 45,9 \\
\hline $\begin{array}{l}\text { 12. Quantas porções de oleaginosas (nozes, amêndoas, incluindo amendoins) consome por } \\
\text { semana? (1 porção: } 30 \mathrm{~g} \text { ) }\end{array}$ & $\geq 3$ & 89,2 & 10,8 \\
\hline $\begin{array}{l}\text { 13. Consome preferencialmente frango, peru ou coelho em vez de vaca, porco, hambúrguer ou } \\
\text { salsicha? }\end{array}$ & Sim & 24,3 & 75,7 \\
\hline $\begin{array}{l}\text { 14. Quantas vezes por semana consome hortícolas, massa, arroz ou outros pratos } \\
\text { confecionados com um refogado (tomate, cebola, alho-francês ou alho e azeite)? }\end{array}$ & $\geq 2$ & 60,5 & 39,5 \\
\hline
\end{tabular}


Associação entre o score de adesão à Dieta Mediterânica e os scores do SF-36 e DHP-18

\begin{tabular}{|c|c|c|c|c|}
\hline & \multicolumn{2}{|c|}{ MODELO NÃO AJUSTADO } & \multicolumn{2}{|c|}{ MODELO AJUSTADO } \\
\hline & B (IC 95\%) & VALOR-p & B (IC 95\%) & VALOR-p \\
\hline \multicolumn{5}{|l|}{ SF-36 } \\
\hline Saúde geral & $2,192(0,841 ; 3,542)$ & $0,002^{*}$ & $2,239(0,871 ; 3,607)$ & $0,001^{*}$ \\
\hline Função física & $3,450(0,932 ; 5,969)$ & $0,008^{*}$ & $3,032(0,886 ; 5,177)$ & $0,006^{*}$ \\
\hline Desempenho físico & $3,427(0,948 ; 5,906)$ & $0,007^{*}$ & $3,160(0,793 ; 5,527)$ & $0,009^{\star}$ \\
\hline Dor física & $2,364(-0,168 ; 4,896)$ & 0,067 & $1,924(-0,606 ; 4,453)$ & 0,135 \\
\hline Desempenho emocional & $1,136(-1,428 ; 3,700)$ & 0,383 & $0,789(-1,793 ; 3,372)$ & 0,547 \\
\hline Função social & $0,044(-2,459 ; 2,548)$ & 0,972 & $-0,386(-2,925 ; 2,153)$ & 0,764 \\
\hline Vitalidade & $1,324(-0,728 ; 3,376)$ & 0,205 & $1,253(-0,714 ; 3,220)$ & 0,210 \\
\hline Saúde mental & $2,390(0,206 ; 4,574)$ & $0,032^{*}$ & $1,615(-0,471 ; 3,701)$ & 0,128 \\
\hline Medida sumário física & $1,718(0,754 ; 2,683)$ & $0,001^{*}$ & $1,696(0,789 ; 2,603)$ & $<0,001^{*}$ \\
\hline Medida sumário mental & $-0,076(-0,954 ; 0,803)$ & 0,865 & $-0,168(-1,094 ; 0,758)$ & 0,721 \\
\hline \multicolumn{5}{|l|}{ DHP-18 } \\
\hline Sofrimento psicológico & $0,110(-0,992 ; 1,213)$ & 0,844 & $0,147(-0,988 ; 1,282)$ & 0,799 \\
\hline Barreiras à atividade & $-1,579(-3,090 ;-0,069)$ & $0,041^{*}$ & $-1,303(-2,854 ; 0,248)$ & 0,099 \\
\hline Alimentação desinibida & $1,147(-0,457 ; 2,751)$ & 0,160 & $1,165(-0,438 ; 2,767)$ & 0,153 \\
\hline
\end{tabular}

Os resultados dos modelos não ajustado e ajustado foram obtidos através da análise de regressão linear simples e múltipla, respetivamente. No modelo ajustado foram incluídas no modelo as seguintes covariantes: idade (anos), género (feminino (GR) e masculino), IMC $\left(\mathrm{kg} / \mathrm{m}^{2}\right)$, A1c (\%), atividade física (<150 minutos por semana ou sedentário/a (GR) e $\geq 150$ minutos por semana ou fisicamente ativo/a), hábitos tabágicos (sim e não (GR)), hipertensão (sim e não (GR)), dislipidemia (sim e não (GR)), nível de escolaridade (ensino básico ou menos (GR), ensino secundário e ensino nivel de escolaridade (ensino básico ou menos (GR), ensino secundário e
superior) e estado civil (solteiro/a, casado/a (GR), divorciado/a e viúvo/a).

\section{DISCUSSÃO DOS RESULTADOS}

Os valores de adesão à DMed encontrados são semelhantes aos descritos anteriormente na população adulta portuguesa ( $9,5 \%$ de boa adesão), por Gregório et al. Os autores depararam-se também com um baixo consumo de azeite, leguminosas, frutas e frutos oleaginosos. Esses grupos de alimentos são os pilares fundamentais por que se rege o padrão alimentar mediterrânico, e estão a ser omitidos da alimentação dos portugueses (16). Neste estudo a amostra apresenta ter excesso ponderal e valores de A1c acima do recomendado para a população diabética $(<7,0 \%)$, principalmente no grupo de indivíduos que tem uma fraca adesão à DMed (17). Segundo os resultados da meta-análise de Huo et al., seria importante adotar uma boa adesão a este padrão alimentar para garantir um melhor controlo glicémico e reduzir o IMC. Os autores explicaram estes factos referindo que a DMed caracteriza-se por um consumo elevado de alimentos de origem vegetal com elevado teor em fibra dietética, alimentos pobres em gorduras saturadas e colesterol, e alimentos com teores elevados de gorduras monoinsaturadas, que em conjunto com outros compostos anti-inflamatórios e antioxidantes presentes nos hortícolas, frutos, cereais integrais e vinho tinto, resultam num melhor controlo do peso corporal e do metabolismo da glucose, e auxiliam a redução do risco cardiovascular (18).

Uma boa adesão à DMed associa-se a uma melhor QdVRS da população diabética principalmente nas dimensões da saúde física: SG (a pessoa acredita que a sua saúde é boa e que não vai piorar); FF (a pessoa consegue realizar todos os tipos de atividade física, incluindo as mais exigentes, sem limitações por motivos de saúde); e DF (a pessoa não tem problemas na realização do trabalho ou de outras atividades diárias em consequência da saúde física) (19). Estes são bons indicadores do estado de saúde daqueles que aderem à Dieta Mediterrânica, e representam um panorama favorável de bem-estar e ausência de incapacidade física.

Na literatura científica, embora uma boa adesão à DMed tenha sido associada a uma melhor qualidade de vida sobretudo nas dimensões da saúde física, tal como neste estudo, outros autores encontraram ainda
* Um valor de $p<0,05$ foi considerado estatisticamente significativo

$\beta$ : Coeficiente beta

A1c: Hemoglobina glicada

GR: Grupo de referência

IMC: Îndice de massa corpora uma associação positiva entre a adesão à DMed e a saúde mental (a pessoa sente-se em paz, feliz e em calma) (20). No entanto, os resultados não foram estatisticamente significativos depois de ajustados para outros fatores, o que também não se verificou no estudo de Pérez-Tasigchana et al., utilizando o PREDIMED como instrumento de avaliação da adesão à DMed (21). Por outro lado, uma boa adesão à DMed parece estar inversamente associada às barreiras à atividade (menos limitações, barreiras e ansiedade ligada às atividades do dia a dia) somente quando não se controlam outras variáveis, o que revela que essa associação não é suficientemente consistente (13). Sendo o primeiro estudo a avaliar a relação entre a adesão à DMed e as dimensões do DHP-18, na conceção de novos estudos seria interessante: usar o DHP-18 como instrumento de mediação da QdVRS; e utilizar amostras de maiores dimensões e boas bases metodológicas que permitam clarificar esta questão.

Como limitações do presente estudo podem-se enumerar as seguintes: 1 - Os participantes foram pesados em balanças diferentes devido à disponibilidade dos gabinetes de avaliação, o que torna variável e impossível de estimar o erro técnico de medição associado à medição do peso corporal; 2 - Não foi aferida a estatura dos participantes por ausência de recursos (estadiómetros) nos diferentes gabinetes de avaliação, tendo-se, por isso, utilizado o valor registado no documento de identificação civil, o qual carece de validação; 3 - Não foram incluídos na análise estatística outros fatores que influenciam a qualidade de vida além da adesão à DMed, como por exemplo, a presença de outras condições patológicas incapacitantes, ou, a ingestão energética diária dos participantes.

\section{CONCLUSÕES}

As pessoas com DM2 têm uma melhor qualidade de vida sobretudo nas dimensões da saúde física, se recorrerem a uma dieta baseada nos princípios da DMed. Em oposição, os indivíduos com fraca adesão à DMed têm piores indicadores de controlo da diabetes, pelo que será necessário investir na divulgação dos benefícios para a saúde deste tipo de dieta junto da comunidade em geral, e, em particular, da população diabética. 


\section{AGRADECIMENTOS}

Muito obrigado a todos os participantes pela disponibilidade, e ao Prof. Doutor Pedro Lopes Ferreira, do Centro de Estudos e Investigação em Saúde da Universidade de Coimbra, por nos ter concedido a versão portuguesa dos questionários de avaliação da QdVRS (SF-36 e Perfil de Saúde do Diabético).

\section{REFERÊNCIAS BIBLIOGRÁFICAS}

1. Serra-Majem L, Trichopoulou A, de la Cruz JN, Cervera P, García Alvarez A, La Vecchia $\mathrm{C}$, et al. Does the definition of the Mediterranean diet need to be updated? Public Health Nutrition. 2004;7(7):927-9.

2. Bach-Faig A, Berry EM, Lairon D, Reguant J, Trichopoulou A, Dernini S, et al Mediterranean diet pyramid today. Science and cultural updates. Public Health Nutrition. 2011;14(12A):2274-84.

3. Sofi F, Cesari F, Abbate R, Gensini GF, Casini A. Adherence to Mediterranean diet and health status: meta-analysis. BMJ. 2008;337:a1344.

4. Sociedade Portuguesa de Diabetologia. Diabetes: Factos e Números 2015 Relatório Anual do Observatório Nacional da Diabetes. [31 de outubro de 2019]. Disponível em: http://www.spd.pt/images/bolsas/dfn2015.pdf.

5. Bonaccio M, Di Castelnuovo A, Bonanni A, Costanzo S, De Lucia F, Pounis G, et al. Adherence to a Mediterranean diet is associated with a better health-related quality of life: a possible role of high dietary antioxidant content. BMJ Open. 2013;3:e003003. 6. Azenha CMC. Qualidade de vida e satisfação com o tratamento em diabéticos tipo II. Coimbra. Dissertação [Mestrado em Gestão e Economia da Saúde] - Faculdade de Economia da Universidade de Coimbra; 2014.

7. Sousa RMCB. Capacitação de pessoas sofrendo de Diabetes tipo 2 em consulta de Medicina Geral e Familiar. Covilhã. Dissertação [Mestrado integrado em Medicina] - Universidade da Beira Interior; 2016.

8. Alcubierre N, Martinez-Alonso M, Valls J, Rubinat E, Traveset A, Hernández M. Relationship of the adherence to the Mediterranean diet with health-related quality of life and treatment satisfaction in patients with type 2 diabetes mellitus: a post-hoc analysis of a cross-sectional study. Health Quality of Life Outcomes. 2016;14:69. 9. Direção-Geral da Saúde. Orientação da Direção-Geral da Saúde nº 017/2013 Avaliação Antropométrica no Adulto. [31 de outubro de 2019]. Disponível em: https:// www.dgs.pt/directrizes-da-dgs/orientacoes-e-circulares-informativas/orientacao-n0172013-de-05122013-pdf.aspx.

10. Martínez-González MÁ, Corella D, Salas-Salvadó J, Ros E, Covas MI, Fiol M et al. Cohort Profile: Design and methods of the PREDIMED study. International Journal of Epidemiology. 2012;41(2):377-85.

11. Afonso L, Moreira T, Oliveira A. Índices de adesão ao padrão alimentar mediterrânico - a base metodológica para estudar a sua relação com a saúde. Revista Fatores de Risco. 2014;31:48-55

12. Ferreira, PL. Criação da versão portuguesa do MOS SF-36 Parte I - Adaptação Cultural e Linguística. Acta Médica Portuguesa 2000;13:55-66.

13. Cruz RS, Leitão CE, Ferreira, PL. Determinantes do estado de saúde dos diabéticos. Revista Portuguesa de Endocrinologia, Diabetes e Metabolismo. 2016;11(2):188-196. 14. Ferreira PL. Criação da versão portuguesa do MOS SF-36. Parte II - Testes de validação. Acta Médica Portuguesa 2000; 13: 119-127.

15. Ferreira PL, Ferreira LN, Pereira LN. Medidas sumário física e mental de estado de saúde para a população portuguesa. Revista Portuguesa de Saúde Pública. 2013;30(2):163-171

16. Gregório MJ, Rodrigues AM, Graça P, de Sousa RD, Dias SS, Branco JC, et al Food Insecurity Is Associated with Low Adherence to the Mediterranean Diet and Adverse Health Conditions in Portuguese Adults. Frontiers in Public Health. 2018;6:38 17. American Diabetes Association. 6. Glycemic Targets: Standards of Medical Care in Diabetes. Diabetes Care. 2018;41(Suppl.1):S55-S64.

18. Huo R, Du T, Xu Y, Xu W, Chen X, Sun K, et al. Effects of Mediterranean-style diet on glycemic control, weight loss and cardiovascular risk factors among type 2 diabetes individuals: a meta-analysis. European Journal of Clinical Nutrition. 2015;69(11):1200-8. 19. Ware JE, Snow KK, Kosinki M, Gandek B. SF-36 Health Survey: Manual and Interpretation Guide. Boston: Whashigton Street; 1993.
20. Henríquez Sánchez P, Ruano C, de Irala J, Ruiz-Canela M, Martínez-González MA, Sánchez-Villegas A. Adherence to the Mediterranean diet and quality of life in the SUN Project. European Journal of Clinical Nutrition. 2012; 66(3):360-8.

21. Pérez-Tasigchana RF, León-Muñoz LM, López-García E, Banegas JR, RodríguezArtalejo F, Guallar-Castillón P. Mediterranean Diet and Health-Related Quality of Life in Two Cohorts of Community-Dwelling Older Adults. PLoS One. 2016;11(3):e0151596. 